\title{
3. Outcome of the Antimicrobial Drug Guideline
}

\author{
Toshiharu MATSUSHIMA
}

Respiratory Center, Kurashiki Daiichi Hospital, Kurashiki

\begin{abstract}
Key words: guideline, pneumonia, antimicrobial chemotherapy, outcome
\end{abstract}

Pneumonia is a serious disease with both a high incidence and mortality. Since the place of its treatment varies, including home, clinics, small hospitals, and regional hospitals, treatment is performed by various physicians, such as family, medical, respiratory, infection, and emergency physicians. There are also various causative microorganisms and antimicrobial drugs. Therefore, pneumonia is treated by a wide variety of methods. If these methods are integrated into one good treatment method, the most effective optimal guideline may be designed. Therefore, since 1991, advanced countries have proposed guidelines. In Japan, the establishment of the guideline was delayed, but a questionnaire survey of the members of the Japanese Respiratory Society and physicians in clinics showed that about $90 \%$ considered an original guideline in Japan to be necessary. Therefore, the Japanese Respiratory Society decided to establish "guidelines for respiratory infection" and proposed a guideline for communityacquired pneumonia in 2000 , one for nosocomial pneumonia in 2002, and one for respiratory tract infection in 2003.

The basic principle for the production of guidelines is shown. This principle accurately describes plans of research, survey, and revision. Pathogenic microorganisms for community-acquired pneumonia were prospectively studied with Prof. Saito of Ryukyu University as the chairman. The subjects were all consecutive patients who visited 7 medical colleges and associated hospitals for the 4-month period between December 2000 and March 2001. A strict protocol was used, and all clinical samples (such as sputum and serum) were sent to medical institutions profficient in the examination of bacteria, chlamydia, mycoplasma, legionella, Q fever coxiella, and viruses. As a result of thorough examination of pathogens, the pathogen could be identified in $70 \%$ of the patients. Streptococcus pneumoniae was the most frequent pathogen, followed in order by Haemophilus influenza, viruses, mycoplasma, and chlamydia; legionella was also a frequently observed pathogen. The incidences of these microorganisms were almost identical to those observed in previous prospective multi-center studies in Japan and also similar to those reported in western countries.

Among studies on the quality of the guideline in regional hospitals, Dr. Kuwabara of Hiroshima Prefectural Hospital compared the conventional treatment and the treatment according to the guideline. He reported a high rate of treatment on an outpatient basis, a high rate of the identification of pathogens, and a high improvement rate after treatment in the group treated according to the guideline, and also suggested that the guideline can be incorporated into clinical path. A study was performed with Dr. Nakahama in Osaka as the chairman, in 20 clinics in Japan. The subjects were 168 patients with community-acquired pneumonia, and the usefulness of the guideline was reported. Dr. Watanabe et al of Tohoku University performed a nationwide study on community-acquired pneumonia. They collected 1,258 cases of pneumonia from 135 institutions and analyzed the disease type, pathologic condition, treatment results, and outcomes and suggested some problems based on the results. Evaluation of the types of antimicrobial drugs and their frequency of use in the 1,258 cases showed use of the new quinolones in only $6 \%$. This result seems fitting because the study was performed according to the guideline, suggesting observance of the principles of the guideline, which showed the limitations in the use of the new quinolones. The frequency of the use of carbapenem was slightly high (15.2\%). However, considering the high percentage of severe pneumonia cases $(23.8 \%)$, limitations in the use of this drug may have also been observed. In general, $\beta$-lactam antimicrobial drugs were frequently used.

After the proposal of the guideline, the number of presentations and papers in academic conferences has been increasing, indicating an increased attention to respiratory infections.

To evaluate how widely the guideline is known at 1,2 , and 3 years after its proposal, a questionnaire survey was given to physicians who attended medical seminars, and academic lectures given by the Japan Medical Association. Fifty-sixty percent of the physicians knew of the presence of the guideline for community-acquired pneumonia while only $15-19 \%$ actually used it. Though an annual increase in the percentage was expected, it decreased. However, $90 \%$ of the physicians considered that a guideline is necessary and hoped to use it. In contrast, almost 100\% of the members of the Japanese Respiratory Society knew about the guideline, and $70 \%$ of them had used it.

Though $90 \%$ of the physicians considered a guideline for the treatment of pneumonia to be necessary, only $50 \%$ of general practitioners as the subjects for the use of the guideline knew of the presence of this guideline, and less than

Reprint requests should be addressed to Dr. Toshiharu Matsushima, Respiratory Center, Kurashiki Daiichi Hospital, 5-3-10 Oimatsu, Kurashiki 710-0862 
$20 \%$ actually used it.

The studies on pathogenic microorganisms for community-acquired pneumonia and the validation of the guideline in hospitals with different characteristics, which were planned at the time of the proposal of the guideline, have been completed, and the results have already been reported. Attention to respiratory infection and the number of presentations and papers in academic conferences has been increasing. However, it is still too early to discuss improvement in treatment results as the original purpose.

\title{
4. The Appropriate Use of Antimicrobial Agents in the Treatment of Infectious Diseases and Guidelines Future Trends, "Global Standard ?" or "Regional Standard ?"
}

\author{
Atsushi SAITO and Futoshi HigA \\ Division of Infectious Diseases, Department of Internal Medicine, Graduate School of Medicine, University of the Ryukyus, Okinawa
}

\begin{abstract}
Key words: infectious diseases, antimicrobial chemotherapy, practical guideline, global standard, regional standard
\end{abstract}

\section{Introduction}

To date, clinical guidelines for the appropriate use of antimicrobial agents in the treatment of infectious diseases have been developed and updated by revisions in western countries (1-8). In Japan, Japanese Respiratory Society (JRS) published several practical guidelines: guideline for the treatment of community acquired pneumonia (9) in 2000 (to be revised in 2004), for the treatment of hospital acquired pneumonia in 2002 (10), and for the treatment of respiratory tract infections in 2003 (11). The guideline for the optimal usage of antimicrobial agents has been developed by the cooperation of the Japanese Society for Chemotherapy and the Japanese Association for Infectious Disease in 2001 (12), which will be revised in 2004 .

The characteristics of guidelines published by Japanese societies and those published in the United States are reviewed here, which may show us the answer to whether we need "global standard" guidelines or "regional standard" guidelines where they are expected to be utilized. Health insurance systems are completely different in Japan and the U.S. The typical dosages and availability of the drugs also sometimes differ. These facts suggest that regional tailored guidelines are desired. In contrast, pharmacokinetics (PK) and pharmacodynamics (PD) of antimicrobials are the universal principles to be considered whenever discussing the optimal usage of antimicrobials. Furthermore, clinical evidence has been collected from all over the world. Hence, it is likely that the "global standard" guidelines which are based on the universal principles and a large quantity of evidence must be more reliable.

\section{Community-acquired pneumonia (CAP) guidelines in the United States as the "global standard"}

In the United States, CAP guidelines have been published by different societies, individually: One by American Thoracic Society (ATS) in 1993 (1), which was revised in 2001 (2); another by Infectious Diseases Society for America (IDSA) in 1998 (4). The latter was updated in 2000 (5) and in 2003 (6). These two guidelines are quite different in the strategies in diagnosis, the categorization of patients, and choice of antimicrobials and sometimes are confusing. Therefore, a CAP guideline is now being developed by cooperation of both ATS and IDSA, which should be a consensus guideline to represent both infectious disease physicians and pulmonary disease physicians and subsequently the guideline will be kind of "global standard".

We need to imagine the "global standard" CAP guideline before it will come out. It is not easy because CAP itself is a very heterogeneous syndrome including various severities, various pathogens, and undetermined factors. However, we can say that the guideline should be based on the huge amount of evidence gathered worldwide. This evidence includes 1) double blinded random therapeutic studies comprised of a huge number of cases, 2) PK/PD indices based on large studies, and 3) the latest data on drug susceptibility of pathogens causing pulmonary infections.

\section{Factors influencing the clinical efficacies of antimicro- bials}

The factors influencing the clinical efficacies of anti-

Reprint requests should be addressed to Dr. Atsushi Saito, Division of Infectious Diseases, Department of Internal Medicine, Graduate School of Medicine, University of the Ryukyus 207 Uehara, Nishihara, Okinawa 903-0215 